\title{
The Cumulation and Dissemination of Knowledge as Preconditions for Effective Crime Prevention Strategies in Forensic Sciences and Sex Research
}

\author{
Martin Rettenberger ${ }^{\text {ab }}$, Mark E. Olver ${ }^{\mathrm{c}}$, L. Maaike Helmus ${ }^{\mathrm{d}}$, Reinhard Eher ${ }^{\mathrm{e}}$ \\ [a] Centre for Criminology (Kriminologische Zentralstelle - KrimZ), Wiesbaden, Germany. [b] Johannes Gutenberg- \\ University Mainz (FGU), Mainz, Germany. [c] University of Saskatchewan, Saskatoon, SK, Canada. [d] Simon Fraser \\ University, Burnaby, BC, Canada. [e] Federal Evaluation Centre for Violent and Sexual Offenders, Austrian Prison \\ System, Vienna, Austria.
}

Sexual Offending: Theory, Research, and Prevention, 2020, Vol. 15(1), Article e3657, https://doi.org/10.5964/sotrap.3657

Published (VoR): 2020-07-22

Corresponding Author: Martin Rettenberger, Centre for Criminology (Kriminologische Zentralstelle - KrimZ), Viktoriastraße 35, D-65189 Wiesbaden, Germany. E-mail: m.rettenberger@krimz.de

As editors of the newly launched journal "Sexual Offending: Theory, Research, and Prevention (SOTRAP)" we are pleased to welcome you to our first issue and we would like at first to thank all colleagues who have and will further support this endeavor. SOTRAP is an international peer-reviewed journal open to all scientists, clinicians, and policymakers researching and preventing all forms of sexual violence. We welcome all contributions that enhance or illuminate relevant clinical practice, science, and policy about the etiology, prevention, (risk) assessment, treatment, and management of individuals who have committed sexual offenses or are at risk of doing so. Furthermore, aspects of legal, psychological, and somatic consequences of sexual offending are of interest for the readership of SOTRAP.

SOTRAP is the official journal of the International Association for the Treatment of Sexual Offenders (IATSO; for more information see www.iatso.org), which was founded on March $24^{\text {th }}, 1998$ in Caracas, Venezuela during an international conference on the treatment of individuals who have committed sexually motivated offenses. IATSO is an international non-profit organization committed to the promotion of research of and treatment for individuals convicted of sexual offenses throughout the world by organizing biennial international conferences, disseminating new research findings and treatment methods, and by providing education and networking opportunities. The aspiration of IATSO is the advocacy of humane, dignified, comprehensive, ethical and 
effective treatment of persons who have committed sexual offenses, in order to contribute to effective crime prevention strategies to reduce further victimizations and human suffering.

Since 2006 IATSO was responsible for the publication of the predecessor of SOTRAP, "Sexual Offender Treatment" (SOT, under its founding editor Reinhard Eher, for more information see www.sexual-offender-treatment.org). We would like to use this opportunity to thank Pabst Science Publishers, who has provided the electronic platform for the publication of the free access research articles of SOT, for the excellent cooperation and support. At the same time, we would like to thank the Leibniz Institute for Psychology Information (ZPID; for more information see https://leibniz-psychology.org/en/) for giving us the opportunity to take the next step and to become part of the PsychOpen GOLD program.

The background for moving the old journal structure to the PsychOpen GOLD program was that it has become apparent for us that the main goals of IATSO and the ZPID - as part of the international open science movement-are an excellent match. Together we are convinced that the dissemination and exchange of knowledge and information are preconditions of implementing effective and ethical crime prevention strategies. However, in many social science disciplines there has been an intense and critical discussion about the stability and generalization of empirical research findings in the last few years. At the same time, we know that scientific evidence is based on replication, and the reproducibility of empirical findings is a key issue in forensic sciences and sex research. These aspects lead inevitably to the conclusion that we should foster every effort to share knowledge and to support our international networks.

Therefore, the SOTRAP editors would like to invite clinicians, practitioners, and academics to submit research articles, reviews, meta-analyses, brief research notes/articles (e.g., brief methodological discussions), and (clinical) case reports/studies to the journal. We accept both qualitative and quantitative research. As stated before, we fully acknowledge the value and importance of open science practices and SOTRAP would like to contribute to a better understanding and implementation of open science practices in the field of sex research and forensic sciences. We are pleased to present the first SOTRAP issue and are looking very much forward to your submissions at sotrap.psychopen.eu

In the moment where we are writing this editorial the world is facing a huge medical challenge and there is a considerable reason to fear that the COVID-19 pandemic and its social, medical, economic, and psychological consequences will accompany us for a long time. In this period of time, which is already extremely difficult for so many of our family members, friends, and colleagues, we have received very sad news that two of our colleagues passed away. Reflecting on the evolution of the journal and the movement towards open science is not possible without also reflecting on the history of our field. Therefore, we would also like to take this opportunity to acknowledge the life and 
professional contributions of two distinguished figures in the field of sexual offending research, who sadly and recently we lost: Ruth E. Mann and D. Richard Laws ${ }^{1}$.

Ruth Mann had a superior ability to identify and operationally define clinically relevant research questions and adapt research to clinical practice. Her work on treatment program development and implementation for those who have sexually offended directly influenced the content of programs around the world. Her work on psychologically meaningful risk factors set a standard for risk assessment. She also researched prison culture and contributed to the more general correctional literature. Fortunately, her considerable intellectual contributions will continue to influence the field for years to come through her many publications in journals and books. However, the best reflection of her impact will be seen through her continued influence on students, trainee psychologists, those she mentored, and front-line providers, to whom she always made herself accessible. Condolence messages from those lucky enough to have worked with Ruth Mann have included words like compassionate, remarkable, role model, smart, effective, nice, admirable, graceful, serene, encouraging, amazing, and influential. Many admitted it was impossible to effectively describe her impact on their careers in just a few short sentences. This is even more true for those of us who were fortunate enough to consider Ruth Mann to be a friend as well as a colleague. We are all immensely grateful that she chose to apply her many talents to our professional area, she will be greatly missed.

Richard Laws started in our field in the 1960s, and since his start, he never stopped and quickly became a major contributor in the field. His impact is easily seen over the decades regarding how we conduct treatment with individuals who sexually offend, ranging from behavioural treatments to relapse prevention, to desistance of sexual offending and historically surveying sexual offender assessment. He easily gained and retained enormous respect and admiration by his peers for his thoughtful contributions, advancing the field and evolving our understanding and treatment of sexual abusers. So, to say he was an icon in our field is an understatement. He had vision, insight, and an unwavering sense of we can and should do better. Richard Laws has been appreciated by so many colleagues, who have been fortunate to meet him personally and worked together with him, and many have been influenced by him as a mentor, a father figure, and a friend. It is indescribably sad that we have lost him, and he will be sorely missed. Richard has left us and our field in a better state than when he entered, and for that we will be forever grateful.

\section{Martin Rettenberger, Mark E. Olver, L. Maaike Helmus, and Reinhard Eher Editors-in-Chief}

1) We would like to thank Yolanda Fernandez, Sandy Jung, and Carmen Zabarauckas for their contribution to the following paragraphs. To read the full tribute to Richard Laws from which we took our excerpts with permission of the authors, see https://blog.atsa.com/2020/04/d-richard-laws.html 
Sexual Offending: Theory, Research, and Prevention is the official journal of the International Association for the Treatment of Sexual Offenders (IATSO).

\section{(P) leibniz-psychology.org}

PsychOpen GOLD is a publishing service by Leibniz Institute for Psychology Information (ZPID), Germany. 